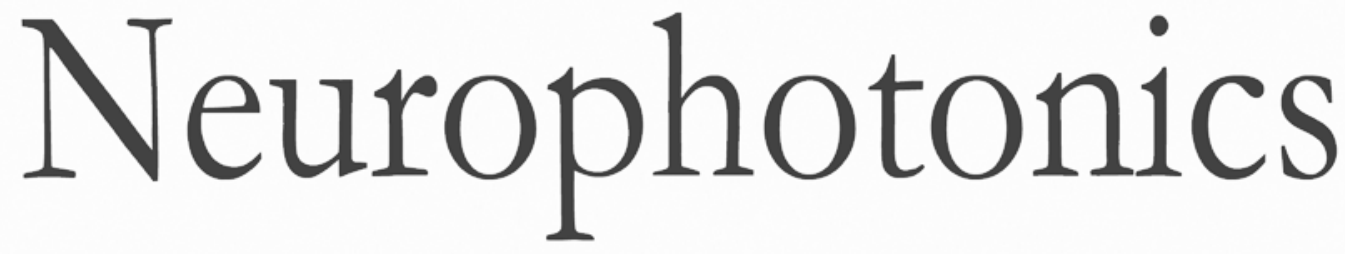

\title{
Functional near-infrared spectroscopy evidence for the development of topological asymmetry between hemispheric brain networks from childhood to adulthood
}

\author{
Lin Cai \\ Qi Dong \\ Mengjing Wang \\ Haijing Niu
}




\title{
Functional near-infrared spectroscopy evidence for the development of topological asymmetry between hemispheric brain networks from childhood to adulthood
}

\author{
Lin Cai, ${ }^{a, b, c}$ Qi Dong, ${ }^{a}$ Mengjing Wang, ${ }^{a, b}$ and Haijing Niu ${ }^{a, b, *}$ \\ ${ }^{a}$ Beijing Normal University, State Key Laboratory of Cognitive Neuroscience and Learning and IDG/McGovern Institute for Brain Research, \\ Beijing, China \\ ${ }^{\mathrm{b}}$ Beijing Normal University, Beijing Key Laboratory of Brain Imaging and Connectomics, Beijing, China \\ ${ }^{c}$ Keio University, Department of Electronics and Electrical Engineering, Yokohama, Japan
}

\begin{abstract}
Cerebral asymmetry is considered an important marker of the successful development of the human brain. Recent studies have demonstrated topological asymmetries between structurally hemispheric networks in the human brain. However, it remains largely unknown whether and how the functionally topological asymmetries evolve from childhood to adulthood, a critical period that constitutes the primary peak of human brain and cognitive development. Here, we adopted resting-state functional near-infrared spectroscopy imaging data to construct hemispheric functional networks and then applied graph theory analysis to quantify the topological characteristics of the hemispheric networks. We found that the adult group exhibited consistent leftward hemispheric asymmetries in both global and local network efficiency, and the degree of leftward asymmetry in local network efficiency was significantly increased with development from childhood to adulthood. At the nodal level, the degree of leftward asymmetry in nodal efficiency, mainly involving the frontal, parietal-occipital junction, and occipital regions, increased with development. These developmental patterns of topological asymmetries suggest that the protracted maturation of functional segregation in the left hemisphere could underlie language development from childhood to adulthood and provide insight into the development of human brain functional networks. ( The Authors. Published by SPIE under a Creative Commons Attribution 4.0 Unported License. Distribution or reproduction of this work in whole or in part requires full attribution of the original publication, including its DOI. [DOI: 10.1117/1.NPh.6.2.025005]
\end{abstract}

Keywords: topological asymmetry; functional networks; connectome; resting-state fNIRS; brain development; lateralization.

Paper 18073RR received Dec. 5, 2018; accepted for publication Apr. 8, 2019; published online May 8, 2019.

\section{Introduction}

Hemispheric asymmetries are fundamental organizational principles of the human brain that are presumed to contribute to fast, efficient information processing, and the specific functions of language and reasoning in humans. ${ }^{1-3}$ Evidence shows that the asymmetries between two brain hemispheres might be altered in states of psychiatric or neurological disease, such as autism spectrum disorder ${ }^{4,5}$ and attention-deficit hyperactivity disorder. ${ }^{6,7}$ Furthermore, healthy individuals also exhibit dynamic changes in brain asymmetries throughout the lifespan. ${ }^{8-12}$ These findings provide valuable information for understanding underlying pathological mechanisms and lateralized cognitive processes, such as language and motor control.

Recently, neuroimaging techniques have been widely applied to human brain network studies. By constructing brain networks within a hemisphere, many studies have demonstrated significant topological asymmetry between hemispheric networks across different age spectra, such as in neonates, ${ }^{13}$ adults, ${ }^{14}$ and elderly participants. ${ }^{15}$ Moreover, from a developmental perspective, Zhong et al. examined the change in structurally topological asymmetry of the hemispheric brain network from adolescence to young adulthood. ${ }^{16}$ The authors found that both adolescents and adults showed rightward asymmetry in network efficiency, and the asymmetry significantly decreased over

*Address all correspondence to Haijing Niu, E-mail: niuhjing@bnu.edu.cn development. Notably, these previous studies primarily adopted structural neuroimaging technique and examined structural asymmetry of hemispheric brain networks. It is known that functional lateralization is putatively associated with structural asymmetries. ${ }^{3}$ However, to date, few studies have reported hemisphere asymmetry in brain functional networks. Moreover, no study has reported how these topological asymmetries of the brain functional network evolve during development.

Here, we utilized resting-state functional near-infrared spectroscopy (rs-fNIRS) imaging data to investigate functional network asymmetry and the development of functional topological asymmetries in children and adults. FNIRS imaging is an emerging cerebral imaging technique that has been widely used to study neural activity in the human brain. ${ }^{17-20}$ By modeling the entire human brain as a network, fNIRS imaging has revealed important topological organization principles underlying the human brain network, e.g., small-worldness, modular organization, and highly connected or centralized hubs. ${ }^{20}$ The graph metrics of fNIRS brain network also exhibited good reliability and validity, ${ }^{21,22}$ suggesting the feasibility of applying fNIRS to the study of the topological asymmetry between hemispheric brain functional networks.

In this study, we sought to determine whether there are topological differences across two brain hemispheres and how the topological asymmetries are distinct between childhood and adulthood, a critical period that constitutes the primary peak of human brain and cognitive development. ${ }^{23-25}$ We hypothesized 
the existence of differences in the functionally topological asymmetry between hemispheric networks. Such differences might underlie the development of specific human cognitive functions, e.g., language ability and spatial working memory, that take place during this period. To test this hypothesis, we constructed hemispheric functional networks in healthy children and adults and then applied graph theoretical approaches to quantify multiple topological measures for the hemispheric networks.

\section{Materials and Methods}

\subsection{Participants}

This study recruited 60 healthy participants, including 30 children (14 males and 16 females, aged 7.0 to 8.9 years, $\mathrm{M} \pm \mathrm{SD}=8.1 \pm 0.6)$ and 30 adults (16 males and 14 females, aged 19 to 27 years, $\mathrm{M} \pm \mathrm{SD}=23.2 \pm 1.9)$. All participants were right-handed according to the Edinburgh Handedness Questionnaire. The participants or their parents gave written informed consent before the initiation of the experiments. Approval for this study was obtained from the Institutional Review Board of the State Key Laboratory of Cognitive Neuroscience and Learning, Beijing Normal University.

\subsection{Data Acquisition}

We used a continuous-wave near-infrared optical imaging instrument (CW6, TechEn Inc., Massachusetts) to measure the time courses of spontaneous changes in oxygenated hemoglobin (oxy-Hb) and deoxygenated hemoglobin (deoxy-Hb). The length of the time course was $\sim 11$ min with a time sampling rate of $50 \mathrm{~Hz}$. Twelve light sources and 24 detectors interlacing at a spatial distance of $3.2 \mathrm{~cm}$ were plugged into a soft but inelastic plastic probe holder and placed on the head of the participants. These source-detector pairs constituted 46 measurement channels covering most cerebral regions of the whole head [Fig. 1(a), left and right panels]. The probe arrays were positioned in reference to the international 10 to 20 coordinate system. Specifically, six detectors below channels 17 to 24 in both hemispheres were set along a coronal line from the vertex to the external auditory pores; thus, their middle point was localized in $\mathrm{Cz}$, and the leftmost and rightmost detectors were fitted around $\mathrm{T} 3$ and $\mathrm{T} 4$, respectively. The spatial separation between adjacent detectors along the bottom of the probe was set to $3 \mathrm{~cm}$, and it was $5.4 \mathrm{~cm}$ between two pieces of probes. As such, the length of $11.4 \mathrm{~cm}(3 \mathrm{~cm}+5.4 \mathrm{~cm}+3 \mathrm{~cm})$ of the whole probe was comparable to a pitch of the 10 to 20 landmarks of the participants' heads. The pitches of the 10 to 20 landmarks at the low of head circumference were estimated to range from $11.4 \mathrm{~cm}$ for a head with $51 \mathrm{~cm}$ (7- to 8-year-old children) to $12.29 \mathrm{~cm}$ for a head with $55 \mathrm{~cm}$ (adults). Thus, the measurement inconsistency across two groups of participants with different head sizes was in the range of $0.89 \mathrm{~cm}$. Optical data from each channel were collected at two different wavelengths (690 and $830 \mathrm{~nm}$ ). For each participant, we evaluated relative changes in oxy- $\mathrm{Hb}$ and deoxy-Hb signals from an arbitrary 0 baseline at the beginning of the measurement period in units of millimolar millimeter, ${ }^{26}$ using the modified Beer-Lambert Law with a differential path-length factor of 6 (more details about the differential path-length factor could be found in Refs. 27-31). For each participant, the rs-fNIRS data were collected for $\sim 11 \mathrm{~min}$, and the participants were instructed to relax, keep their eyes closed, and remain awake.

\subsection{MRI Coregistration}

To validate the positioning of the probes, a structural magnetic resonance image (MRI) was acquired from one arbitrarily selected adult subject. During MRI data acquisition, the participant lay supine with the probe arrays in an MRI scanner. Vitamin E capsules were attached to each of the optode locations in the probe arrays and were used as landmarks for coregistration [Fig. 1(a), middle panel]. T1-weighted structural MRIs were acquired using a magnetization-prepared rapid gradient echo (MPRAGE) sequence with a 3T Siemens Tim Trio MRI scanner in the Imaging Center for Brain Research, Beijing Normal University. Scanning parameters were as follows: 176 slices, repetition time $(\mathrm{TR})=2600 \mathrm{~ms}$, echo time $(\mathrm{TE})=$ $3.02 \mathrm{~ms}$, field of view $(\mathrm{FOV})=256 \times 224 \mathrm{~mm}^{2}$, voxel size $=$ $1 \mathrm{~mm} \times 1 \mathrm{~mm} \times 1 \mathrm{~mm}$, flip angle $=8 \mathrm{deg}$, and slice orientation $=$ sagittal. The MRIs were normalized into Montreal Neurological Institute (MNI) space using the NIRS_SPM software, ${ }^{32}$ and the MNI coordinates for each measurement channel were determined according to the automated anatomical labeling (AAL) template. ${ }^{33}$ A similar positioning method was used in previous fNIRS studies. ${ }^{34-36}$ The cortical position corresponding to each measurement channel is shown in Table S1 in the Supplementary Materials.

\subsection{Data Preprocessing}

Data preprocessing was conducted using the in-house FC-NIRS package (Ref. 37), which was developed using MATLAB 2010b in a 64-bit Windows 7 environment. After data preprocessing, the optical density signal was transformed to the time courses of spontaneous changes of hemoglobin concentration (i.e., oxy-Hb and deoxy-Hb and total-Hb) using modified Beer-Lambert law. Of note, the hemoglobin concentration signal was band-pass filtered in the frequency range of 0 to $3 \mathrm{~Hz}$.

\subsection{Noise and Artifact Removal via ICA}

For the time courses of spontaneous changes in oxy-Hb and deoxy-Hb, we first conducted a temporal independent component analysis (ICA) to remove the typical motion-induced artifacts and systematic physiological noise. ${ }^{19,22}$ This procedure was performed using publicly available software, FastICA $\mathrm{v} 2.5,{ }^{38}$ and more details could be found in several previous rs-fNIRS studies. ${ }^{19,22,39,40}$ Specifically, we identified typical noise components (e.g., motion artifacts and systematic physiological interference) according to the components' temporal profiles, spatial maps, and power spectra. A component would be considered noise if it met one of the following conditions: ${ }^{22}$ (1) the corresponding temporal profile included sudden jumps, slowly varied U or inverted U-shaped spike, or numerous intercurrent quick spikes (e.g., motion artifacts); (2) the dominant frequency of power spectra of the component was outside the range of 0.01 to $0.1 \mathrm{~Hz}$; (3) the spatial map of the component presented a global and spatially dispersive pattern (e.g., physiological interference). The noise components, e.g., motion and systematic physiological noise, were visually identified for each participant. After identifying these types of noise components, each concentration signal was reconstructed with these particular components eliminated 
(a)
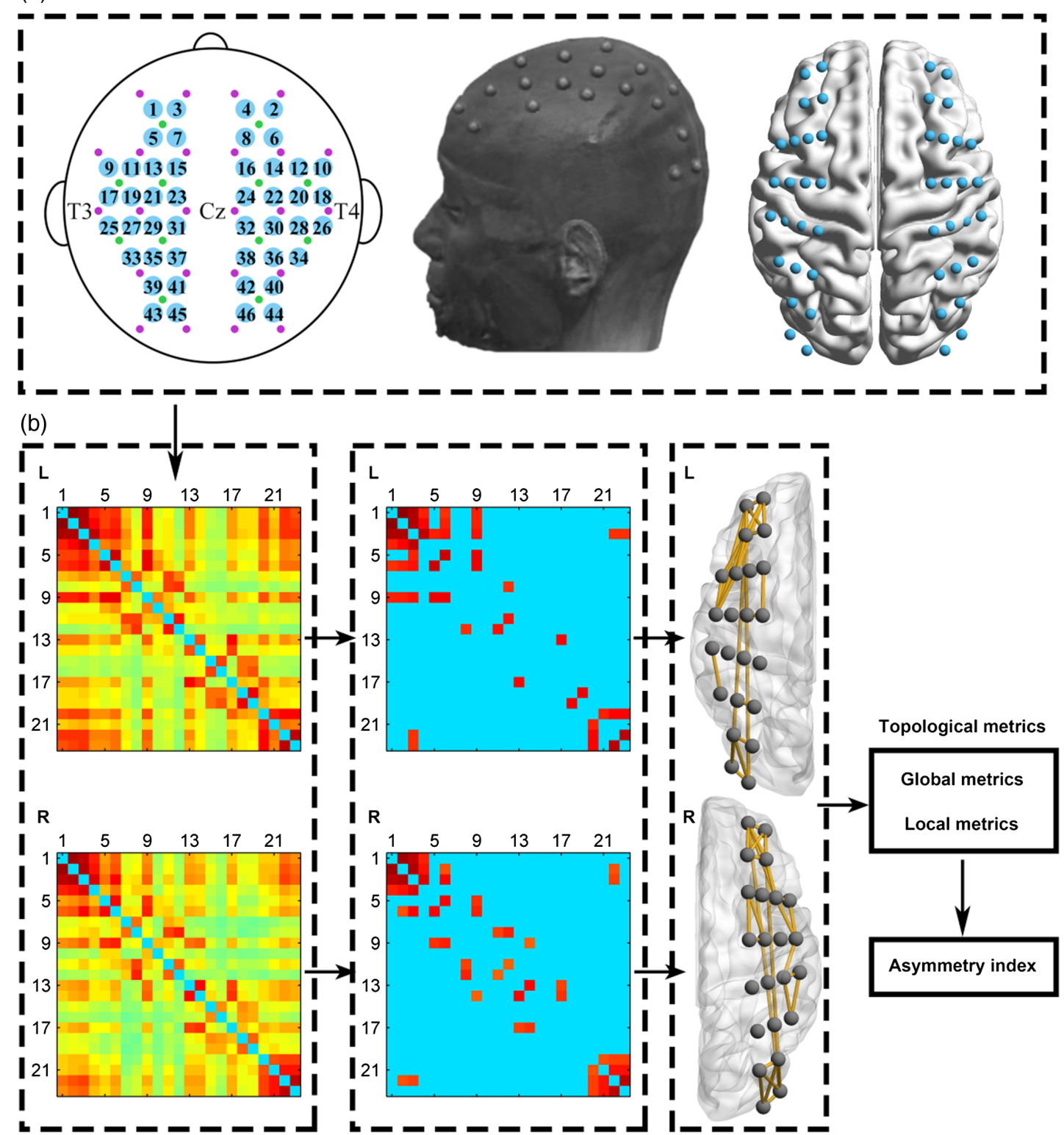

Fig. 1 (a) fNIRS data collection and MRI neuroanatomical coregistration. The left panel shows the arrangement of the 46 measurement channels across the entire head. The green and purple dots represent the sources and detectors, respectively. The digits represent the positions of the measurement channels. The middle panel shows that MRI coregistration was conducted by having the participant wear probe arrays with vitamin E capsules during the MRI scan. The right panel shows the anatomical position corresponding to each measurement channel. (b) Flowchart for the procedure used to construct the hemispheric brain functional networks.

from the original rs-fNIRS time course by replacing zero in the corresponding column of mixing matrix. ${ }^{40}$ To obtain the low-frequency hemodynamic signals that emanated from spontaneous neural activity and to reduce the effect of high-frequency noise and baseline drift, ${ }^{35,41,42}$ we conducted a bandpass filter with cutoff frequencies of 0.009 and $0.08 \mathrm{~Hz}$ on the ICA denoised time series. Figure 2 showed a direct examples of representative time traces before and after ICA as well as after band-pass filtering. Notably, after ICA and band-pass filtering $(0.009$ to $0.08 \mathrm{~Hz})$, the noise with sudden jumps and slow-varied U-shaped spikes were removed. Finally, we extracted same amount of data, i.e., 10-min continuous time course, for all participants. The data were then used to conduct functional connectivity analyses and network topology analyses.

\subsection{Construction of the Left and Right Hemispheric Networks}

We used MATLAB functions from the GRETNA toolbox ${ }^{43}$ to construct two $23 \times 23$ symmetric correlation matrices, one for the left hemisphere and the other for the right hemisphere. Similarly, the correlation coefficient was calculated using the Pearson correlation method. Due to the ambiguous biological explanation of negative correlations in the correlation matrix, ${ }^{44,45}$ we set all negative correlation coefficients to zero 

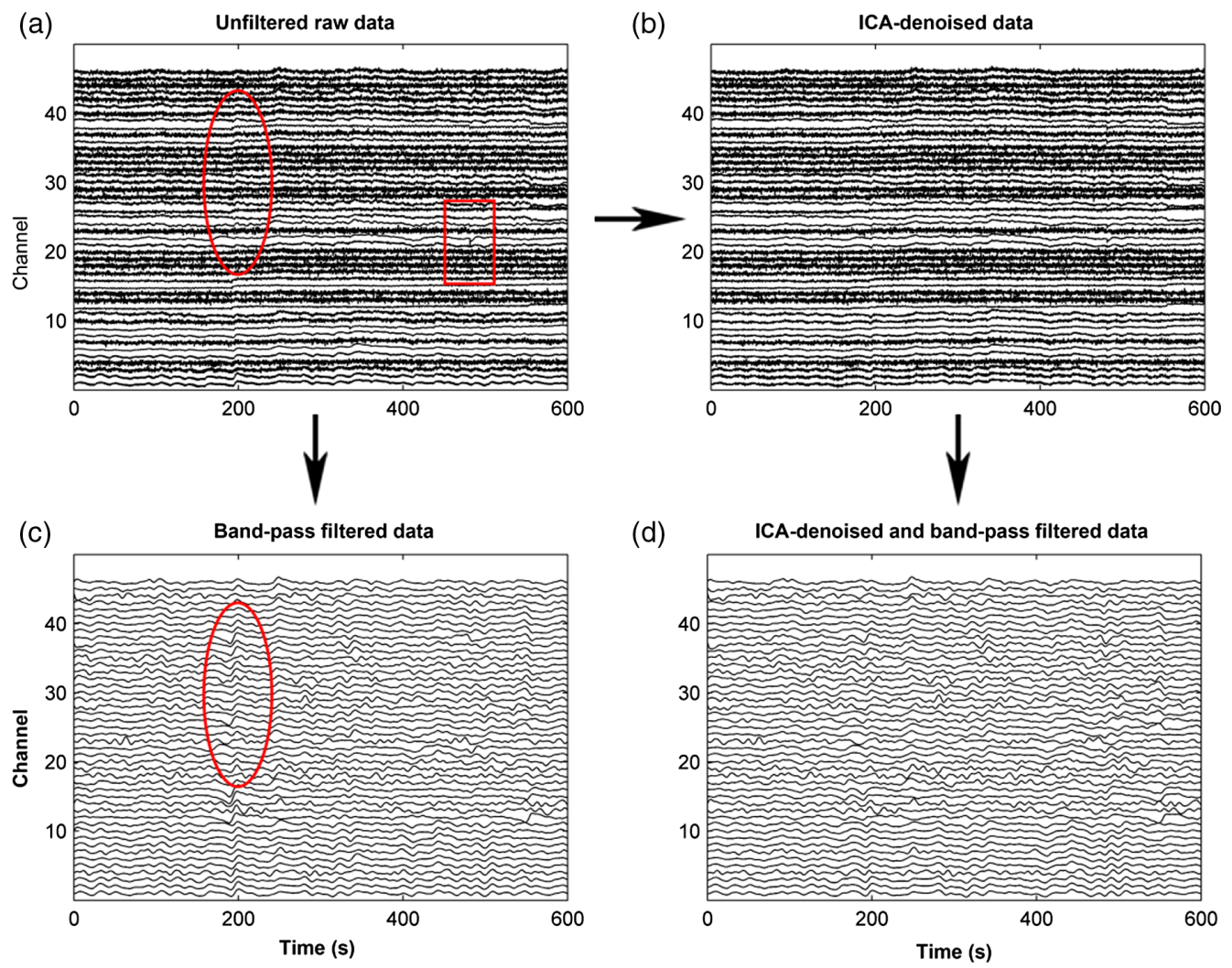

Fig. 2 The fNIRS signals before and after ICA filtering. (a) The raw fNIRS signals (oxy-Hb). For convenient visual inspection, the raw rs-fNIRS data were detrended using the first and second polynomial functions and then transformed into $z$-scores via subtracting the mean value and dividing by standard deviation for each channel. A red ellipse indicates slow-varied U-shaped spikes and a red rectangle indicates sudden jumps occurred in one channel. (b) The fNIRS signals after ICA filtering. Notably, the noise with sudden jumps and slow-varied U-shaped spikes were removed after ICA filtering. (c) The fNIRS signals after band-pass filtering. Notably, the noise with sudden jumps was removed, but the noise with slow-varied U-shaped spikes could not be removed using the traditional bandpass filtering (as indicated by the red ellipse). (d) The fNIRS signals after ICA and band-pass filtering.

and restricted our analysis to positive correlations. These correlation coefficients were then converted to $z$-values via Fisher's $r$-to- $z$ transformation to improve the normality. Each correlation matrix was thresholded to a weighted matrix with a fixed sparsity value (defined as the total number of edges in a network divided by the maximum possible number of edges) and retaining the suprathreshold weights. This sparsity-based thresholding method ensured that the hemispheric networks in each age group had the same number of edges and wiring cost. ${ }^{46,47}$ Therefore, a range of sparsity (i.e., 0.1 to 0.2 ) with an interval of 0.01 was selected for graph theoretical analysis of the hemispheric functional brain network to maintain the reachability of the network and allow prominent small-world properties. To provide a threshold-independent comparison between groups in network properties, we calculated the area under curve (AUC, i.e., the integral) for each network metric from sparsity 0.1 to 0.2 with an interval of 0.01 . These AUC values were used to perform further statistical analyses. ${ }^{48}$

\subsection{Network Analysis}

Graph theory has been used to characterize the topological organization of human brain functional networks, proving a powerful and quantitative way to examine the segregation and integration of brain networks. ${ }^{49}$ To examine the small-world attributes of a network $\mathrm{G}$ that consisted of $N$ nodes and $K$ edges, the normalized global efficiency (the normalized $E_{\text {glob }}=E_{\text {glob }}^{\text {real }} / E_{\text {glob }}^{\text {rand }}$ ) and the normalized local efficiency (the normalized $\left.E_{\mathrm{loc}}=E_{\mathrm{loc}}^{\text {real }} / E_{\mathrm{loc}}^{\mathrm{rand}}\right)$ were computed. ${ }^{50} E_{\mathrm{glob}}^{\text {real }}$ and $E_{\text {loc }}^{\text {real }}$ are the global efficiency and local efficiency of a real network, respectively, and $E_{\mathrm{glob}}^{\text {rand }}$ and $E_{\mathrm{loc}}^{\text {rand }}$ represent the means of the corresponding parameters derived from 100 matched random networks that have the same numbers of nodes, edges, and distribution of degrees as the real brain network. Typically, a small-world network should meet the following criteria: the normalized $E_{\mathrm{glob}} \approx 1$ and the normalized $E_{\mathrm{loc}} \gg 1^{51}$

\subsubsection{Global efficiency}

The global efficiency $E_{\text {glob }}$ of network $G$ is a global measure of the information transferring ability of the entire network and is defined as the inverse of the harmonic mean of the shortest path length between any two nodes: ${ }^{.2}$

$E_{\mathrm{glob}}=\frac{1}{N(N-1)} \sum_{i \neq j \in G} \frac{1}{d_{i j}}$,

where $d_{i j}$ is the shortest path length between node $i$ and node $j$. The shortest path length was the minimum number of edges 
included in the path that connected these two nodes. $N$ denoted the number of nodes of the network $G$.

\subsubsection{Local efficiency}

The local efficiency $E_{\text {loc }}$ of network $G$ is defined as the average of the local efficiencies of all nodes, where the local nodal efficiency for a given node $i$ is the global efficiency of the subgraph composed of the nearest neighbors to node $i: 47,52$

$E_{\mathrm{loc}}=\frac{1}{N} \sum_{i \in G} E_{\mathrm{glob}}(i)$,

where $E_{\text {glob }}(i)$ is the global efficiency of $G_{i}$, which is the subgraph of the neighbors of node $i$.

\subsubsection{Nodal efficiency}

Nodal efficiency represents the capacity of a node to communicate with the other nodes of a network, and the efficiency of node $i$ is measured as follows:

$E_{\mathrm{nod}}(i)=\frac{1}{N-1} \sum_{j \neq i \in G} \frac{1}{d_{i j}}$,

where $d_{i j}$ is the shortest path length between node $i$ and node $j$. Nodes with a high nodal efficiency indicate that the network has a high tolerance for the elimination of a given node, which is associated with a high clustering of the neighborhood of this node. $^{47}$

\subsection{Asymmetry Index}

To characterize the degree of asymmetry for efficiency measures (i.e., global efficiency, local efficiency, and nodal efficiency), we calculated the asymmetry index (AI) using the following equation:

$\mathrm{AI}=\frac{\mathrm{ML}-\mathrm{MR}}{\mathrm{ML}+\mathrm{MR}}$

where ML represents measures in the left hemisphere, and MR represents measures in the right hemisphere. For example, for the AI calculation on network efficiency, ML and MR represent the global or local efficiency of the left and right hemispheric network, respectively. For the AI of nodal efficiencies, ML and MR represent the nodal efficiency of corresponding channels in the left and right hemispheric networks, respectively. Thus, AI ranges from -1 to +1 , with a positive value of $\mathrm{AI}$ representing a leftward asymmetry and vice versa.

\subsection{Statistical Analysis}

Hemispheric asymmetry of topological organization has been suggested to be associated with gender. ${ }^{15,53}$ Thus, we first evaluated whether gender had an influence on topological organization using a univariate analysis of covariance (ANCOVA) with group (children and adults) as a between-subject factor, hemisphere (left and right) as a repeated-measures factor, and gender (male and female) as a covariate. However, the "gender $\times$ hemisphere" interaction was not significant for any of the efficiency parameters. Therefore, we excluded gender and performed a two-way mixed-design analysis of variance (ANOVA) with group (children and adults) as a between-subject factor and hemisphere (left and right) as a repeated-measures factor. If any main effect survived a threshold of $p<0.05$, a further $t$ test (paired $t$-test for the hemisphere effect and two-sample $t$ test for the group effect) was performed. Moreover, to assess age-related effects on the AI of the network's global efficiency, local efficiency, and nodal efficiency, we performed a two-tailed two-sample $t$-test on the AI of each parameter. For global/local efficiency, $p<0.05$ was considered significant. For nodal efficiency comparison between two hemispheres (23 nodes in each hemisphere) in each age group, the false discovery rate (FDR) procedure was applied to correct for multiple comparisons, ${ }^{54}$ and $q<0.05$ was considered significant. In addition, when comparing the AI of nodal efficiency between two age groups (23 AI values for each age group), FDR was also applied to correct the multiple comparisons, and $q<0.05$ was considered significant.

\section{Results}

\subsection{Group Differences of Hemispheric Functional Connectivity}

Figure 3 showed the group-averaged hemispheric functional connectivity for both children and adults, respectively. For each participant, the sparsity thresholds from 0.1 to 0.2 with an interval of 0.01 were used to construct hemispheric brain network. For left hemispheric network, the edge-density thresholds of 0.1 and 0.2 corresponded to the strength of functional connectivity (i.e., Pearson- $r$ values) of 0.66 (sparsity $=0.2$ ) and 0.78 (sparsity $=0.1$ ) for adults, and 0.58 (sparsity $=0.2$ ) and 0.68 (sparsity $=0.1$ ) for children, respectively. For right hemispheric network, such edge-density thresholds corresponded to Pearson- $r$ values of 0.61 (sparsity $=0.2$ ) and

(a) Children(Left hemisphere)

(b) Adults(Left hemisphere)
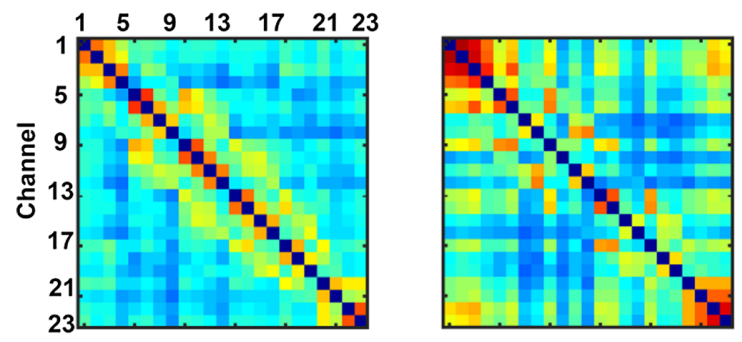

(c) Children(Right hemisphere) (d) Adults(Right hemisphere)

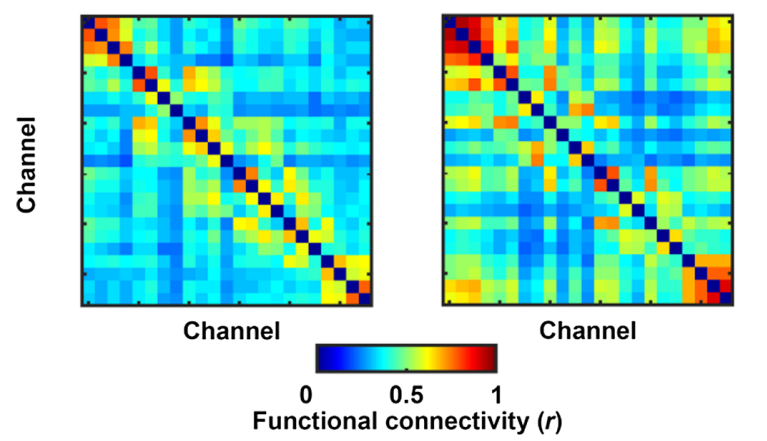

Fig. 3 Group-averaged hemispheric functional connectivity matrices for both children and adults, respectively. Digits in functional connectivity matrices represent 23 measurement channels for each hemisphere. 
0.73 (sparsity $=0.1)$ for adults, and 0.55 (sparsity $=0.2$ ) and 0.67 (sparsity $=0.1$ ) for children, respectively.

The group comparisons in functional connectivity (i.e., Pearson- $r$ ) were calculated at three chosen threshold values (e.g., 0.1, 0.15, and 0.2) using two sample $t$-tests, respectively. For left hemispheric network, we found no significant difference at sparsity $=0.2(p>0.05)$ and significant differences at sparsity $=0.1$ and $0.15(p<0.05)$, respectively. For right hemispheric network, we found no significant differences at three different sparsity threshold values. These results demonstrated asymmetry of the development in hemispheric functional connectivity from children to adults.

\subsection{Economic Small-World Organization}

Small-world topology in each hemispheric network was observed across both children and adults. As shown in Fig. 4, for each hemispheric functional network, the normalized $E_{\text {loc }}$ was larger than 1 , and the normalized $E_{\text {glob }}$ was nearly 1 across these two age groups. This finding indicates that the hemispheric networks exhibited prominent small-world properties, consistent with previous whole-brain fNIRS network studies. ${ }^{19}$ The small-world organization principle within hemispheric functional networks suggests that each cerebral hemisphere maintains a balance between local segregation and global integration for both children and adults.

\subsection{Within-Group Asymmetry of Global and Local Network Efficiency}

The global and local network efficiencies were calculated using an integrated threshold (i.e., the AUC, including sparsity from 0.1 to 0.2 with an interval of 0.01 ). For global network efficiency, a significant hemisphere main effect was observed (a) Children(Left hemisphere)

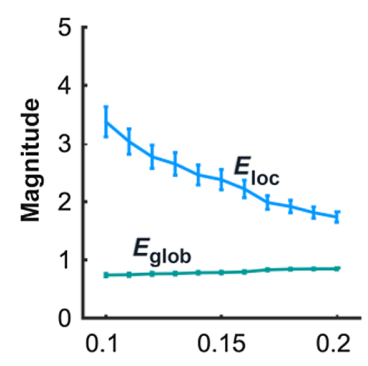

(c) Children(Right hemisphere)

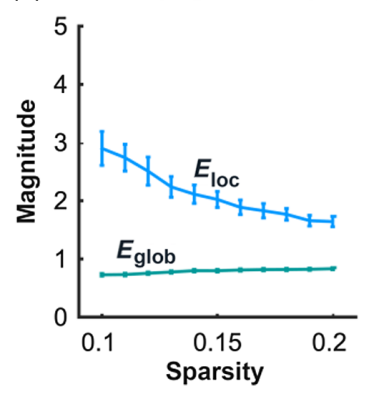

(b) Adults(Left hemisphere)

(d) Adults(Right hemisphere)

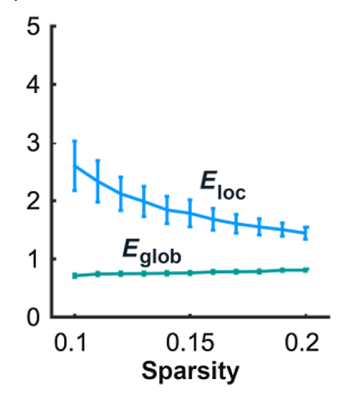

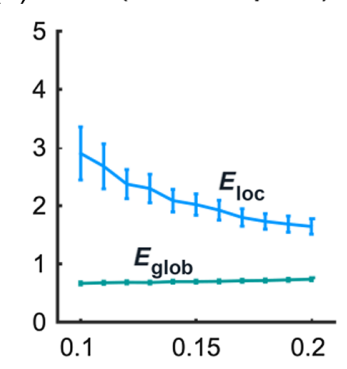

Fig. 4 Small-world characteristics of the hemispheric functional networks for children and adults. The graphs show the changes in normalized global and local efficiency as a function of sparsity thresholds. Error bars indicate the standard errors of all participants in each subgroup.

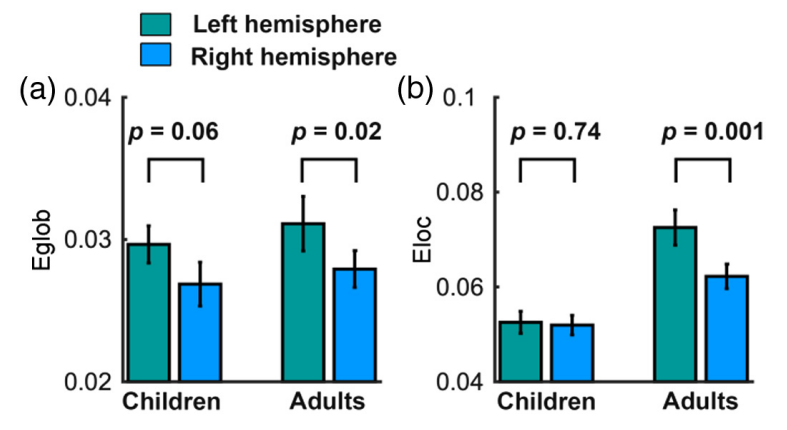

Fig. 5 Within-group asymmetry of the global and local network efficiency. (a) Global efficiency ( $\left.E_{\text {glob }}\right)$ and (b) local efficiency $\left(E_{\text {loc }}\right)$.

$[F(1,58)=9.76 ; p=0.003]$. Further paired $t$-test analysis revealed significant leftward asymmetry in adults $[t=2.51$, $p<0.05$; Fig. 5(a)] and a trend toward leftward asymmetry in children $[t=1.95, p=0.06$; Fig. 5(a)]. However, for local efficiency, both the significant hemisphere main effect $[F(1,58)=10.43 ; p=0.002]$ and hemisphere $\times$ group interaction effect $[F(1,58)=8.34 ; p=0.005]$ were significant. Further paired $t$-test analysis suggested that only the adult group showed significant leftward hemispheric asymmetry $[t=3.57$, $p=0.001$, for adults; $t=0.33, p=0.74$, for children, Fig. 5(b)].

\subsection{Between-Group Differences in the Asymmetry of Global and Local Network Efficiency}

Two-sample $t$-tests revealed that there was no significant difference in the hemispheric asymmetry of the global efficiency between groups [children: $\mathrm{AI}=0.06 \pm 0.03$; adults: $\mathrm{AI}=0.05 \pm 0.02 ; t=0.35, p=0.73$, Fig. 6(a)]. However, (a)
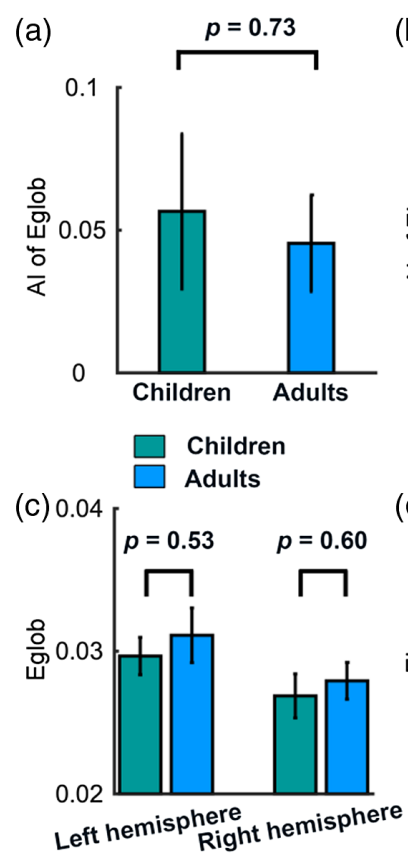

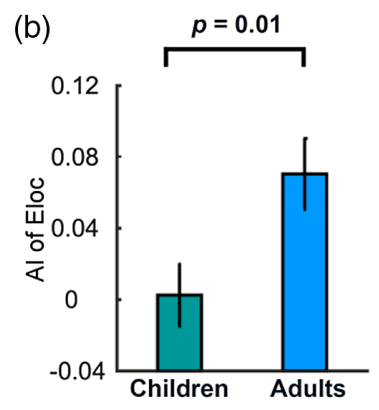

(d) 0.1

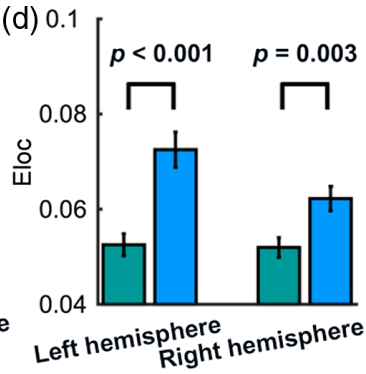

Fig. 6 Between-group differences in the Al of the global and local network efficiency. (a) Global efficiency $\left(E_{\text {glob }}\right)$ and (b) local efficiency $\left(E_{\mathrm{loc}}\right)$. Group comparison within each hemisphere in (c) global efficiency $\left(E_{\text {glob }}\right)$ and $(\mathrm{d})$ local efficiency $\left(E_{\mathrm{loc}}\right)$. 
for local efficiency, the hemispheric asymmetry showed a significant increase over development [children: $\mathrm{AI}=0.00 \pm 0.02$; adults: $\mathrm{AI}=0.07 \pm 0.02 ; t=-2.57, p=0.01$, Fig. 6(b)]. Post hoc analysis using a two-sample $t$-test within hemisphere showed no significant age effect on global efficiency in the left $(t=-0.63, p=0.53)$ or right hemisphere $[t=-0.53$, $p=0.60$, Fig. 6(c)]. However, a significant increase in local efficiency was observed from childhood to adulthood in both the left $(t=-4.57, \quad p<0.001)$ and right hemispheres $[t=-3.12, p=0.003$, Fig. 6(d)].

\subsection{Within-Group Asymmetry of Nodal Efficiency}

For each group, the group-averaged map of nodal efficiency is shown in Fig. 7(a). Visual inspection suggested that the spatial patterns of the mean nodal efficiency were very similar across these two hemispheres. Quantitatively, for each group, the linear correlation analysis across all nodes revealed a very high correlation between the two hemispheres [Fig. 7(b), $r=0.82$, $p<0.0001$, for children; $r=0.90, p<0.0001$, for adults]. Figure 7(c) shows the hemispheric network nodes that showed significant between-hemisphere differences in nodal efficiency (uncorrected $p<0.05$ ). For each of these two groups, primarily leftward asymmetry was observed, but the proportion of leftward asymmetry was obviously higher $(17.4 \%)$ in adults than in children (8.7\%) [Fig. 7(c)]. For children, the positions of these asymmetries were mainly located in the frontal region (channels 7 to 8 and 23 to 24); for adults, the positions of these asymmetries were mainly located in the frontal (channels 19 to 20) and parietal (channels 35 to 38 ) regions and outside of the occipital (channels 39 to 40) regions. The adult group only showed a rightward asymmetric node near the occipital region (channel 41/42).

\subsection{Between-Group Differences in the Asymmetry of Nodal Efficiency}

The significant group difference in the AI of nodal efficiency is shown in Fig. 8(a). Over development, some nodes showed leftward asymmetry (e.g., red spheres), and these nodes were primarily distributed in the frontal, parietal-occipital junction, and occipital regions (channels 17, 18 to $35,36,39$, and 40) [uncorrected $p<0.05$, Fig. 8(a)]. Some nodes showed that children displayed leftward asymmetry, whereas adults displayed rightward asymmetry (blue spheres), and these nodes were mainly located in the frontal regions associated with the default network and the frontal-parietal control network [channels 15 and 16; uncorrected $p<0.05$, Fig. 8(a)]. Overall, these nodes showed less rightward asymmetry in the adults than in the children. For each hemisphere, the average nodal efficiency based on these significant left-lateralized and right-lateralized nodes did not show any significant differences between the two age groups [Figs. 8(b) for significant left-lateralized nodes and Fig. 8(c) for significant right-lateralized nodes, respectively].
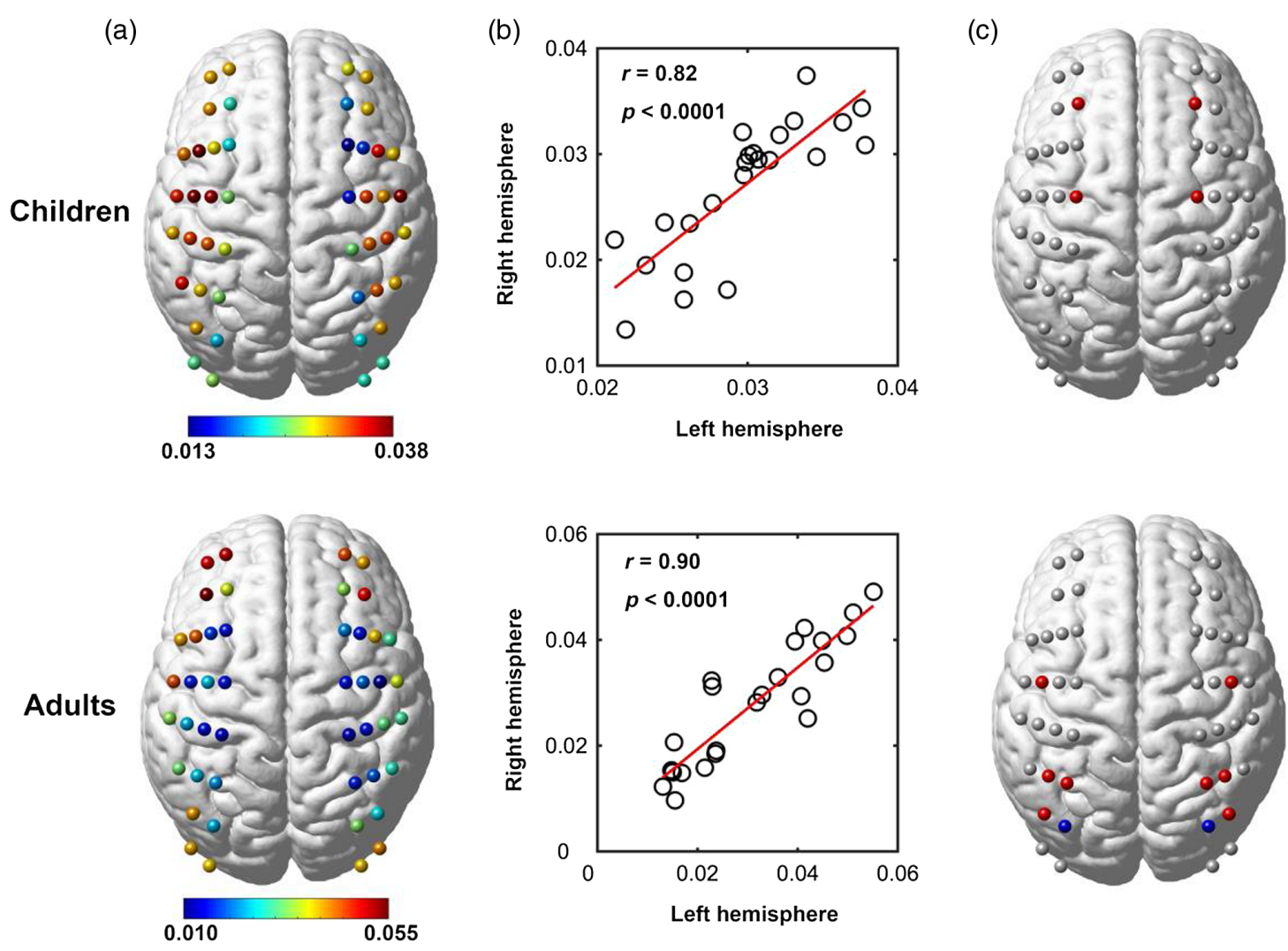

Fig. 7 Within-group asymmetry in the nodal efficiency across the brain. (a) The group-averaged nodal efficiency for the two hemispheric networks was projected onto the cortical surface. Color represents the mean efficiency values across all subjects, with larger values indicating higher information processing ability for the nodal regions, vice versa. (b) The between-hemisphere correlation of the mean nodal efficiency across all nodes. Each circle represents a node within the hemisphere. (c) Statistically significant nodal efficiency asymmetry for children and adults. The red spheres indicate the significant left-lateralized positions, and the blue spheres indicate the significant right-lateralized positions. 
(a)

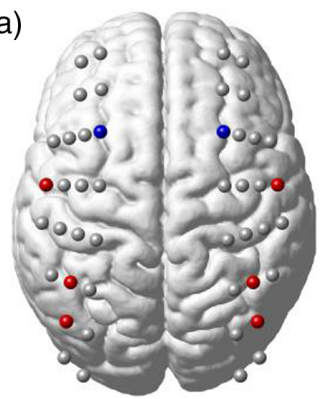

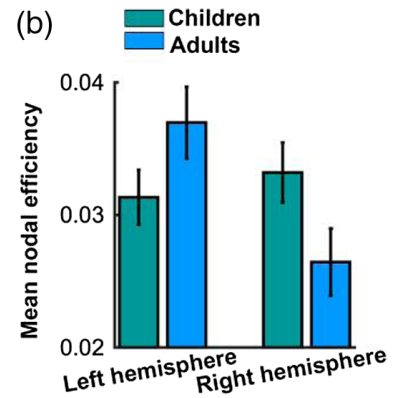

(c)

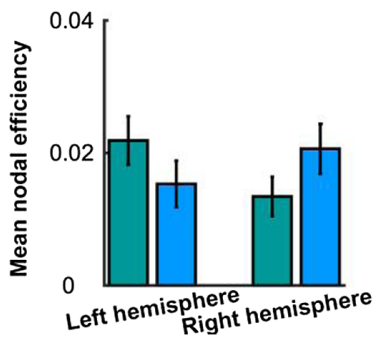

Fig. 8 (a) Between-group differences in the Al of nodal efficiency. The red spheres indicate the significant left-lateralized positions, and the blue spheres indicate the significant right-lateralized positions. (b) Group comparison in the mean nodal efficiency in the left-lateralized nodes (i.e., red spheres) within each hemisphere. (c) Group comparison in the mean nodal efficiency in the right-lateralized nodes (i.e., blue sphere) within each hemisphere.

\section{Discussion}

In this study, using rs-fNIRS techniques and graph theory analysis, we investigated the development of the hemispheric asymmetry of functional networks from childhood to adulthood. At a hemispheric level, we provided the first report of brain functionally topological asymmetry associated with development. Using an fNIRS imaging technique, we revealed efficient topological architecture within both right and left hemispheric networks for children and adults, respectively. We found leftward asymmetry (or trend) in hemispheric network efficiency and noted that the degree of leftward hemispheric asymmetry in network local efficiency significantly increased with development. The findings suggest that the protracted maturation of functional segregation in the left hemisphere may underlie language development from childhood to adulthood and provide insight into the development of human brain functional networks. These findings are discussed in greater detail below.

\subsection{Small-Worldness of Hemispheric Functional Networks}

The small-worldness architecture supporting efficient information segregation and integration with low energy and wiring cost advances our understanding of the topological organization of complex brain networks. ${ }^{55-59}$ In recent studies, a small-world organization has been consistently observed in the wholebrain structural and functional networks across different age spectra. ${ }^{55,60-62}$ In this study, at a hemispheric scale, we also observed that the brain was organized into a small-worldness organization across both children and adults, suggesting that each cerebral hemisphere, similar to the whole-brain network architecture, is also organized in an efficient and compact manner to preserve fast information segregation and integration. Notably, this fNIRS finding is also consistent with previous functional and structural MRI studies exploring brain asymmetry between hemispheric networks. ${ }^{13-15,63}$ Collectively, this accumulated evidence demonstrates that small-world topology is a fundamental principle of the organization of brain networks. Therefore, our observations extend these earlier findings and provided new evidence to indicate that the brain in childhood maintains a balance between local segregation and global integration within each hemisphere.

\subsection{Hemispheric Asymmetries in Brain Functional Networks through Childhood and Adulthood}

With a functional optical imaging technique, we found that the hemispheric brain also showed leftward asymmetries (or trends) in hemispheric network efficiency, which indicates that the left hemisphere in children and adults exhibited more efficient functional segregation and integration than the right hemisphere to adapt to left-lateralized functional needs. The current findings are consistent with prior observations from structural network studies of hemispheric asymmetry. ${ }^{13,15,53,63,64}$ For instance, Ratnarajah et al. ${ }^{13}$ found that the neonate brain showed greater global and local efficiency in the left hemisphere than those in the right, demonstrating that such left-lateralized efficient topological organization plays crucial roles in specific lateralized brain functions (e.g., motor, memory, language functions) at birth. Dennis et al. ${ }^{64}$ found that the global efficiency was greater in the left hemisphere in early adulthood. Caeyenberghs and Leemans ${ }^{15}$ reported that the left hemisphere was significantly more efficient than the right hemisphere from young adulthood to old age. Sun et al. ${ }^{63}$ found that older adults (60 to 82 years) showed a leftward asymmetry in both global and local efficiency. However, there are several results ${ }^{53,63}$ inconsistent with these current lateralization findings, demonstrating symmetric functional network topology or rightward lateralization in network efficiency. These contrasting findings may be attributed to the age range of the subject sample, various imaging methods, network construction methods, and network complexity.

In line with the well-documented leftward asymmetry in language, motor, and visual functions, ${ }^{65}$ our findings revealed brain regions with significant leftward asymmetry in nodal efficiency in frontal (channels 7 to 8 and 23 to 24 for children, channels 19 to 20 for adults) and parietal-occipital junction (channels 35 to 38 for adults) regions and in regions outside of the occipital (channels 39 to 40 for adults) regions, indicating that these lateralized cerebral regions exhibited more efficient communications in the left hemisphere than the right homologues in the right hemisphere. Therefore, the leftward asymmetry in motor and language functions observed in most righthanded people ${ }^{66}$ allowed us to speculate that these leftlateralized brain regions identified in the current study may contribute to a more integrated network topology in the left hemisphere. 


\subsection{Developmental Effects on the Topological Asymmetry of the Functional Network}

In this study, we found that the degree of leftward asymmetry in network global efficiency is statistically equivalent between children and adults [Fig. 6(a)]. These findings are supported by the lack of observed developmental effects in network global efficiency in each hemisphere [Fig. 6(c)]. In line with these observations, previous studies of the development of wholebrain functional networks demonstrated no significant difference in network global efficiency across development. ${ }^{67-69}$ Notably, compared with children, adults showed significantly increased leftward asymmetry in local network efficiency [Fig. 6(b)]. Specifically, our data showed a larger developmental increase in local efficiency in the left hemisphere than in the right hemisphere [Fig. 6(d)]. The protracted maturation of functional segregation in the left hemisphere may underlie language development from childhood to adulthood. Many studies have demonstrated that the greatest asymmetries of structure are clearly localized to the perisylvian language area (see a review, Ref. 3). For example, Szaflarski and his colleagues ${ }^{70}$ found that language lateralization to the left hemisphere increases between the ages of 5 and 20 years and plateaus between 20 and 25 years, which reveals that there might be hemispheric differences in white matter maturation, perhaps during the many regional growth spurts in myelination that occur in childhood. ${ }^{71}$ Except for the development of language, the maturation of other cognitive functions is likely associated with the degree of left lateralization. For instance, a leftward asymmetry in paracingulate cortex thickness was related to better spatial working memory in healthy subjects aged 16 to 51 years. ${ }^{72}$ Therefore, our observed dramatic developmental changes in leftward asymmetries in network local efficiency likely suggest that the left hemisphere plays a leading role in highly demanding cognitive processes, such as language and memory, which may require more segregated functional subnetworks across development. Moreover, although Zhong et al. ${ }^{16}$ found the degree of rightward asymmetry in both global and local network efficiencies significantly decreased from adolescence to young adulthood, these developmental patterns in network asymmetry may, to some extent, reflect the rapid development of the left hemisphere in charge of language.

\subsection{Further Considerations}

There are some potential limitations of the current study. First, this study did not investigate the relationship between network asymmetry and behavioral measurements. In the future, measurements involving language abilities and social cognitive evaluation are expected to further consolidate the primary findings in this study, such as the development of left lateralization in network local efficiency from childhood to adulthood. Second, the present results were based on a cross-sectional dataset; follow-up, longitudinal developmental dataset is highly desired to validate the current findings. Third, we utilized the same probe geometry for data collection in both child and adult participants. Head circumference constitutes an important index in the characterization of brain development for children. Fourth, we did not record the level of motion of all participants during the stage of data acquisition, therefore, motion components were purely visually identified for all analyses. In the future, a more objective or automatic component identification strategy should be adopted, such as template-matching. Finally, our study contained a relatively small sample size, and future work should enroll more subjects and investigate how handedness and gender factors modulate the hemispheric topological asymmetry.

\section{Conclusion}

In sum, we provided new evidence to indicate that the brain in childhood keeps a balance between local segregation and global integration within two hemispheres, like that in the adult brain. For network global and local efficiency, we found that children exhibited relatively symmetric network efficiency, while adults showed significant leftward asymmetry. Notably, the degree of leftward asymmetry in network local efficiency showed a significant increase from childhood to adulthood. The developmental patterns of topological asymmetries suggested that the protracted maturation of functional segregation in the left hemisphere may underlie language development from children to adults and provided insight into the development of human brain functional networks.

\section{Disclosure}

The authors have declared that no conflict of interest exists.

\section{Acknowledgments}

This study was supported by the Natural Science Foundation of China (Grant nos. 81571755, 81761148026, and 31521063). We wish to thank Ms. Shujie Geng for helping with data acquisition and Ms. Jingyu Wang for data analyses.

\section{References}

1. J. Levy, "Possible basis for the evolution of lateral specialization of the human brain," Nature 224(5219), 614-615 (1969).

2. H. S. Liu et al., "Evidence from intrinsic activity that asymmetry of the human brain is controlled by multiple factors," Proc. Natl. Acad. Sci. U. S. A. 106(48), 20499-20503 (2009).

3. A. W. Toga and P. M. Thompson, "Mapping brain asymmetry," Nat. Rev. Neurosci. 4(1), 37-48 (2003).

4. P. R. Escalante-Mead, N. J. Minshew, and J. A. Sweeney, "Abnormal brain lateralization in high-functioning autism," J. Autism Dev. Disord. 33(5), 539-543 (2003).

5. N. M. Kleinhans et al., "Abnormal functional connectivity in autism spectrum disorders during face processing," Brain 131, 1000-1012 (2008).

6. L. M. Foster et al., "Planum temporale asymmetry and ear advantage in dichotic listening in developmental dyslexia and attention-deficit/hyperactivity disorder (ADHD)," J. Int. Neuropsychol. Soc. 8(1), 22-36 (2002).

7. G. W. Schrimsher et al., "Caudate nucleus volume asymmetry predicts attention-deficit hyperactivity disorder (ADHD) symptomatology in children," J. Child Neurol. 17(12), 877-884 (2002).

8. G. Li et al., "Spatial patterns, longitudinal development, and hemispheric asymmetries of cortical thickness in infants from birth to 2 years of age," J. Neurosci. 35(24), 9150-9162 (2015).

9. D. M. Zhou et al., "Cortical thickness asymmetry from childhood to older adulthood," Neuroimage 83, 66-74 (2013).

10. N. Paquette et al., "Developmental patterns of expressive language hemispheric lateralization in children, adolescents and adults using functional near-infrared spectroscopy," Neuropsychologia 68, 117-125 (2015).

11. O. Agcaoglu et al., "Lateralization of resting state networks and relationship to age and gender," Neuroimage 104, 310-325 (2015).

12. T. Arimitsu et al., "Functional hemispheric specialization in processing phonemic and prosodic auditory changes in neonates," Front. Psychol. 2, 202 (2011).

13. N. Ratnarajah et al., "Structural connectivity asymmetry in the neonatal brain," Neuroimage 75, 187-194 (2013). 
14. Y. Iturria-Medina et al., "Brain hemispheric structural efficiency and interconnectivity rightward asymmetry in human and nonhuman primates," Cereb. Cortex 21(1), 56-67 (2011).

15. K. Caeyenberghs and A. Leemans, "Hemispheric lateralization of topological organization in structural brain networks," Hum. Brain Mapp. 35(9), 4944-4957 (2014).

16. S. Y. Zhong et al., "Developmental changes in topological asymmetry between hemispheric brain white matter networks from adolescence to young adulthood," Cereb. Cortex 27(4), 2560-2570 (2017).

17. G. Boas, "Noninvasive imaging of the brain," Opt. Photonics News 15(1), 52-55 (2004).

18. F. F. Jobsis, "Noninvasive, infrared monitoring of cerebral and myocardial oxygen sufficiency and circulatory parameters," Science 198(4323), 1264-1267 (1977).

19. L. Cai, Q. Dong, and H. J. Niu, "The development of functional network organization in early childhood and early adolescence: a resting-state fNIRS study," Dev. Cognit. Neurosci. 30, 223-235 (2018).

20. H. J. Niu and Y. He, "Resting-state functional brain connectivity: lessons from functional near-infrared spectroscopy," Neuroscientist 20(2), 173-188 (2014).

21. H. J. Niu et al., "Revealing topological organization of human brain functional networks with resting-state functional near infrared spectroscopy," PLoS One 7(9), e45771 (2012).

22. H. J. Niu et al., "Test-retest reliability of graph metrics in functional brain networks: a resting-state fNIRS study," PLoS One 8(9), e72425 (2013).

23. T. Paus et al., "Structural maturation of neural pathways in children and adolescents: in vivo study," Science 283(5409), 1908-1911 (1999).

24. E. R. Sowell et al., "In vivo evidence for post-adolescent brain maturation in frontal and striatal regions," Nat. Neurosci. 2(10), 859-861 (1999).

25. S. J. Blakemore, "The social brain in adolescence," Nat. Rev. Neurosci. 9(4), 267-277 (2008).

26. A. Maki et al., "Spatial and temporal analysis of human motor activity using noninvasive NIR topography," Med. Phys. 22(12), 1997-2005 (1995).

27. M. Cope et al., "Methods of quantitating cerebral near infrared spectroscopy data," Adv. Exp. Med. Biol. 222, 183-189 (1988).

28. D. T. Delpy et al., "Estimation of optical pathlength through tissue from direct time of flight measurement," Phys. Med. Biol. 33(12), 1433-1442 (1988).

29. A. Sassaroli and S. Fantini, "Comment on the modified Beer-Lambert law for scattering media," Phys. Med. Biol. 49(14), N255-N257 (2004).

30. L. Kocsis, P. Herman, and A. Eke, "The modified Beer-Lambert law revisited," Phys. Med. Biol. 51(5), N91-N98 (2006).

31. G. Strangman, M. A. Franceschini, and D. A. Boas, "Factors affecting the accuracy of near-infrared spectroscopy concentration calculations for focal changes in oxygenation parameters," Neuroimage 18(4), 865-879 (2003).

32. J. Chul et al., "NIRS-SPM: Statistical parametric mapping for nearinfrared spectroscopy," Neuroimage 44(2), 428-447 (2009).

33. N. Tzourio-Mazoyer et al., "Automated anatomical labeling of activations in SPM using a macroscopic anatomical parcellation of the MNI MRI single-subject brain," Neuroimage 15(1), 273-289 (2002).

34. I. Kovelman et al., "Dual language use in sign-speech bimodal bilinguals: fNIRS brain-imaging evidence," Brain Lang. 109(2-3), 112123 (2009).

35. S. Sasai et al., "A NIRS-fMRI study of resting state network," Neuroimage 63(1), 179-193 (2012).

36. I. Kovelman et al., "Shining new light on the brain's 'bilingual signature': a functional near infrared spectroscopy investigation of semantic processing," Neuroimage 39(3), 1457-1471 (2008).

37. J. P. Xu et al., "FC-NIRS: a functional connectivity analysis tool for near-infrared spectroscopy data," Biomed Res. Int. 2015, 1-11 (2015).

38. A. Hyvarinen, "Fast and robust fixed-point algorithms for independent component analysis," IEEE Trans. Neural Networks 10(3), 626-634 (1999).

39. H. Zhang et al., "Test-retest assessment of independent component analysis-derived resting-state functional connectivity based on functional near-infrared spectroscopy," Neuroimage 55(2), 607-615 (2011).

40. H. Zhang et al., "Functional connectivity as revealed by independent component analysis of resting-state fNIRS measurements," Neuroimage 51(3), 1150-1161 (2010).
41. B. R. White et al., "Resting-state functional connectivity in the human brain revealed with diffuse optical tomography," Neuroimage 47(1), 148-156 (2009).

42. B. Biswal et al., "Functional connectivity in the motor cortex of resting human brain using echo-planar MRI," Magn. Reson. Med. 34(4), 537-541 (1995).

43. J. H. Wang et al., "GRETNA: a graph theoretical network analysis toolbox for imaging connectomics," Front. Hum. Neurosci. 9, 386 (2015).

44. K. Murphy et al., "The impact of global signal regression on resting state correlations: are anti-correlated networks introduced?" Neuroimage 44(3), 893-905 (2009).

45. M. D. Fox et al., "The global signal and observed anticorrelated resting state brain networks," J. Neurophysiol. 101(6), 3270-3283 (2009).

46. C. J. Stam et al., "Small-world networks and functional connectivity in Alzheimer's disease," Cereb. Cortex 17(1), 92-99 (2007).

47. S. Achard and E. T. Bullmore, "Efficiency and cost of economical brain functional networks," PLoS Comput. Biol. 3(2), e17 (2007).

48. H. Huang et al., "Development of human brain structural networks through infancy and childhood," Cereb. Cortex 25(5), 1389-1404 (2015).

49. O. Sporns, "The human connectome: origins and challenges," Neuroimage 80, 53-61 (2013).

50. D. J. Watts and S. H. Strogatz, "Collective dynamics of 'small-world' networks," Nature 393(6684), 440-442 (1998).

51. T. Uehara et al., "Efficiency of a 'small-world' brain network depends on consciousness level: a resting-state fMRI study," Cereb. Cortex 24(6), 1529-1539 (2014).

52. V. Latora and M. Marchiori, "Efficient behavior of small-world networks," Phys. Rev. Lett. 87(19), 198701 (2001).

53. L. X. Tian et al., "Hemisphere- and gender-related differences in smallworld brain networks: a resting-state functional MRI study," Neuroimage 54(1), 191-202 (2011).

54. Y. Benjamini and Y. Hochberg, "Controlling the false discovery rate: a practical and powerful approach to multiple testing," J. R. Stat. Soc. B 57(1), 289-300 (1995).

55. E. T. Bullmore and O. Sporns, "Complex brain networks: graph theoretical analysis of structural and functional systems," Nat. Rev. Neurosci. 10(3), 186-198 (2009).

56. E. T. Bullmore and O. Sporns, "The economy of brain network organization," Nat. Rev. Neurosci. 13(5), 336-349 (2012).

57. O. Sporns, "The human connectome: a complex network," Ann. N. Y. Acad. Sci. 1224, 109-125 (2011).

58. Y. He and A. Evans, "Graph theoretical modeling of brain connectivity," Curr. Opin. Neurol. 23(4), 341-350 (2010).

59. X. H. Liao, A. V. Vasilakos, and Y. He, "Small-world human brain networks: perspectives and challenges," Neurosci. Biobehav. Rev. 77, 286-300 (2017).

60. M. Cao et al., "Toward developmental connectomics of the human brain," Front. Neuroanat. 10, 17 (2016).

61. G. Collin and M. P. van den Heuvel, "The ontogeny of the human connectome: development and dynamic changes of brain connectivity across the life span," Neuroscientist 19(6), 616-628 (2013).

62. W. Gao et al., "Functional connectivity of the infant human brain: plastic and modifiable," Neuroscientist 23(2), 169-184 (2017).

63. Y. Sun et al., "Asymmetry of hemispheric network topology reveals dissociable processes between functional and structural brain connectome in community-living elders," Front. Aging Neurosci. 9, 361 (2017).

64. E. L. Dennis et al., "Development of brain structural connectivity between ages 12 and 30: a 4-Tesla diffusion imaging study in 439 adolescents and adults," Neuroimage 64, 671-684 (2013).

65. M. M. Mesulam, "From sensation to cognition," Brain 121, 1013-1052 (1998).

66. L. M. Balsamo, B. Xu, and W. D. Gaillard, "Language lateralization and the role of the fusiform gyrus in semantic processing in young children," Neuroimage 31(3), 1306-1314 (2006).

67. K. Wu et al., "Topological organization of functional brain networks in healthy children: differences in relation to age, sex, and intelligence," PLoS One 8(2), e55347 (2013).

68. K. Supekar, M. Musen, and V. Menon, "Development of large-scale functional brain networks in children," PLoS Biol. 7(7), e1000157 (2009).

69. M. Cao et al., "Topological organization of the human brain functional connectome across the lifespan," Dev. Cognit. Neurosci. 7, 76-93 (2014). 
70. J. P. Szaflarski et al., "fMRI study of language lateralization in children and adults," Human brain mapping 27(3), 202-212 (2006).

71. P. M. Thompson et al., "Growth patterns in the developing brain detected by using continuum mechanical tensor maps," Nature 404(6774), 190-193 (2000).

72. A. Fornito et al., "Variability of the paracingulate sulcus and morphometry of the medial frontal cortex: associations with cortical thickness, surface area, volume, and sulcal depth," Hum. Brain Mapp. 29(2), 222-236 (2008)

Lin Cai is a PhD candidate in the Department of Electronics and Electrical Engineering, Keio University. He studied cognitive neuroscience at Peking University and Beijing Normal University and received his MSc degree from Peking University. His work focuses on cognitive development of infants and light propagation in infant head models.

Qi Dong is a professor of the State Key Lab of Cognitive Neuroscience and Learning at Beijing Normal University. He studied psychology at Beijing Normal University, China, and received his PhD in psychology from Beijing Normal University in 1988. His research interests are child development and second language learning.

Mengjing Wang received her bachelor's degree in biomedical engineering from the Central South University in 2017. She received her postgraduate degree from the State Key Laboratory of Cognitive Neuroscience and Learning and IDG/McGovern Institute for Brain Research, Beijing Normal University. Her research interests are using fNIRS combined with graph theory to explore mental diseases, such as attention-deficit hyperactivity disorder and Alzheimer's disease.

Haijing Niu received her MS and PhD degrees in optics from Tianjin University and Beijing Normal University, followed by postdoctoral training in biomedical optics at the University of Texas at Arlington. She is an associate professor of the State Key Lab of Cognitive Neuroscience and Learning at Beijing Normal University, China. She focuses on resting-state fNIRS (rs-fNIRS) imaging studies, e.g., using rs-fNIRS data to study the human brain connectome, the software development of the rs-fNIRS data analysis, and the corresponding applications in both healthy brain development and brain diseases. 